\title{
A possible quantum basis of panpsychism
}

\author{
Gao Shan \\ 11-10, NO.10 Building, \\ YueTan XiJie DongLi, XiCheng District \\ Beijing 100045, P.R.China \\ E-mail: gaoshan.iqm@263.net
}

\section{Introduction}

As we know, the explanation of consciousness presents a uniquely hard problem for science ( Chalmers, 1996 ). It forces us to reexamine the physical and metaphysical foundations of present scientific world view. This retriggers the debate about the long-standing dilemma of panpsychism versus emergentism. It is generally accepted that an essential separation of consciousness and matter will preclude any real integration of consciousness with the present scientific picture of the physical world, and panpsychism and emergentism are the only two main positions that can complete the integration ( Seager et al, 2001 ). Then we must decide whether and how consciousness emerges from mere matter or whether consciousness is a fundamental property of matter.

Undoubtedly panpsychism provides an attracting and promising way to fit consciousness into the scientific picture, according to which is consciousness is a fundamental feature of the world which exists throughout the universe ( Seager et al, 2001 ). The main arguments for panpsychism include the genetic ones, the analogical ones, the intrinsic nature ones and some sort of methodological ones. As we think, although we can't directly confirm panpsychism by empirical test presently, we may see it in some indirect empirical facts through logical microscope. In this paper, we will present a new argument based on revised quantum dynamics ( Ghiradi et al, 1986; Pearle, 1989; Diosi, 1989; Ghiradi et al, 1990; Penrose, 1996; Gao, 1999a, 1999b; Gao, 2000 ), which is generally taken as the promising complete version of present quantum theory ( Penrose, 1989; 1994 ), and has been used to construct a consciousness theory ( Hameroff et al, 1996a; 1996b ). It is shown that consciousness may help to distinguish the nonorthogonal single states in the framework of revised quantum dynamics, while the usual physical measuring device can't ( Gao, 1999c; Gao, 2000 ). We further analyze the possible inferences of this unusual conclusion, and demonstrate that it strongly implies that the physical world is not causally closed without consciousness. The fact that consciousness violates the basic physical principle also reveals that consciousness is a fundamental property of matter, and a complete theory of matter must involve consciousness. We conclude that the above argument provides a possible quantum basis for panpsychism.

\section{The existence of consciousness can be tested using a quantum method}

It is well known that quantum theory is the most basic physical theory in our times, and it provides us the deepest understanding about the physical world. But $\mathrm{s}$ to the evolution of the wave function during measurement, present quantum theory provides by no means a complete description, and the projection postulate is just amakeshift (Bell, 1987 ). It is generally expected that a complete quantum theory should describe the projection or collapse as a dynamical process of wave function, and provide a unified evolution law of the wave function (Bell, 1987; Penrose, 1989; 1994 ). The 
resulting theory is well known as revised quantum dynamics, and is widely and deeply studied recently ( Ghiradi et al, 1986; Pearle, 1989; Diosi, 1989; Ghiradi et al, 1990; Penrose, 1996; Gao, 1999a, 1999b; Gao, 2000 ), in which the linear evolution equation of the wave function is replaced by a stochastic nonlinear equation. At the present time, even if the last complete theory has not been found, but one thing is certain for the revised quantum dynamics, i.e. the collapse process of wave function is one kind of objective dynamical process, and it will take a finite time interval to finish. The following analysis will only rely on this common character of revised quantum dynamics.

As we know, present quantum theory doesn't permit the nonorthogonal single states be distinguished. Moreover, in the framework of revised quantum dynamics, the usual measurement using physical measuring device can't distinguish the nonorthogonal single states either. But when the physical measuring device is replaced by a conscious being and considering the influence of consciousness, it can be shown that the nonorthogonal single states can be distinguished in principle in the framework of revised quantum dynamics ( Gao, 1999c; Gao, 2000 ). Thus the distinguishability of nonorthogonal single states provides one kind of physical method to test the existence of consciousness. This is an unusual conclusion. Here we will reformulate the main ideas.

We let the states to be distinguished are the following nonorthogonal single states $\psi_{0}$ and $\psi_{0}+\psi_{1}$, and the initial perception state of the observer is $\chi$. Then after interaction the corresponding entangled state of the whole system is respectively $\psi_{0} \chi_{0}$ and $\psi_{0} \chi_{0}+\psi_{1} \chi_{1}$, where $\chi_{0}$ and $\chi_{1}$ is respectively the perception state of the observer for the states $\psi_{0}$ and $\psi_{1}$. We assume that the perception time of the observer for the definite state $\psi_{0} \chi_{0}$, which is denoted by $t_{P}$, is shorter than the dynamical collapse time for the superposition state $\psi_{0} \chi_{0}+\psi_{1} \chi_{1}$, which is denoted by $t_{C}{ }^{1}$, and the time difference $\Delta t=t_{C}-t_{P}$ is large enough for the observer to identify. Then the observer can perceive the measured state $\psi_{0}$ or his own state $\chi_{0}$ after time interval $t_{P}$, while for the measured superposition state $\psi_{0}+\psi_{1}$, only after the time interval $t_{C}$ can the observer perceive the collapse state $\psi_{0}$ or $\psi_{1}$, or his own corresponding state $\chi_{0}$ or $\chi_{1}$. Since the observer can also be conscious of the time difference between $t_{P}$ and $t_{C}$, he can distinguish the measured nonorthogonal single states $\psi_{0}$ and $\psi_{0}+\psi_{1}$.

It should be noted that the validity of the above method relies on an apparently unusual condition,

\footnotetext{
${ }^{1}$ It should be noted that, since the collapse time of a single superposition state is an essentially stochastic variable, which average value is $t_{c}$, we should consider the stochastic distribution of the collapse time in a strict sense, i.e. a small number of single states is needed for practical application. In the following discussions, we always simply take the collapse time as the average value $t_{c}$ unless state otherwise.
} 
i.e. the perception time of the conscious being for the definite state is shorter than the dynamical collapse time, or his perception time for the superposition state, and the time difference is long enough for him to identify. It is shown that this condition can be satisfied in principle, and some evidences have indicated that our human being may satisfy this condition ( Duane et al, 1965; Grinberg-Zylberbaum et al, 1994 ). Thus he existence of consciousness can be tested in principle using the above quantum method.

\section{The physical world is not causally closed without consciousness}

It is widely argued that the physical world is causally closed, and the consciousness property assigned by the panpsychism must lack all causal efficacies, i.e. there is a purely physical explanation for the occurrence of every physical event and these explanations don't refer to any consciousness property ( McGinn, 1999 ). On the other hand, if panpsychism is true, then it is reasonably expected that the fundamental consciousness property should take part in the physical world's causal chains, and present itself in our investigation of the physical world. In the following, we will further analyze the possible implications of the above quantum method testing the existence of consciousness. Using a simple example, we will show that the physical world is not causally closed without consciousness, and the explanation for the occurrence of some physical events must refer to the fundamental consciousness property.

As we know, the collapse result of wave function in the usual measurement using physical measuring device is fundamentally random, and the results distribution is determined by the measured wave function. We assume the measured state is $\psi_{0}+\psi_{1}$, the initial state of the physical measuring device is $\varphi$. After interaction the resulting entangled state of the whole system is $\psi_{0} \varphi_{0}+\psi_{1} \varphi_{1}$, and the result state of the physical device after collapse will assume $\varphi_{0}$ or $\varphi_{1}$ with the same probability in a completely random way. Here we define a simple rule, i.e. let the output of the device be numbers 0 and 1 for the input states $\psi_{0}$ and $\psi_{1}$ respectively. Then the output of the device will be a random series of 0 and 1 with the same distribution probability $1 / 2$ after measuring a large number of input states $\psi_{0}+\psi_{1}$.

Now we assume the state $\psi_{0}+\psi_{1}$ is input to a conscious being who satisfies the above unusual condition, for example, the input state $\psi_{0}+\psi_{1}$ is a superposition state of a small number of photons, and it enters the eyes of the conscious being ( Ghirardi, 1999; Thaheld, 2000 ) Let the initial perception state of the conscious being be $\chi$, then after interaction the resulting entangled state of the whole system is $\psi_{0} \chi_{0}+\psi_{1} \chi_{1}$, where $\chi_{0}$ and $\chi_{1}$ is respectively the perception state of the conscious being for the states $\psi_{0}$ and $\psi_{1}$. Since the conscious being satisfies the above condition, he can distinguish the input states $\psi_{0}$ or $\psi_{1}$ and $\psi_{0}+\psi_{1}$. Then when the input state is $\psi_{0}+\psi_{1}$, 
the conscious being is able to know that the input state is not the state $\psi_{0}$ or $\psi_{1}$, for which the rule restricts the output as number 0 or 1 , thus the output for the superposition state $\psi_{0}+\psi_{1}$ can be determined by the freewill of the conscious being, and this doesn't break the rule. Evidently such physical events can't be explained without referring to consciousness. This proves that the physical world is not causally closed without consciousness.

The above demonstration can be clearly depicted in the following black box system:

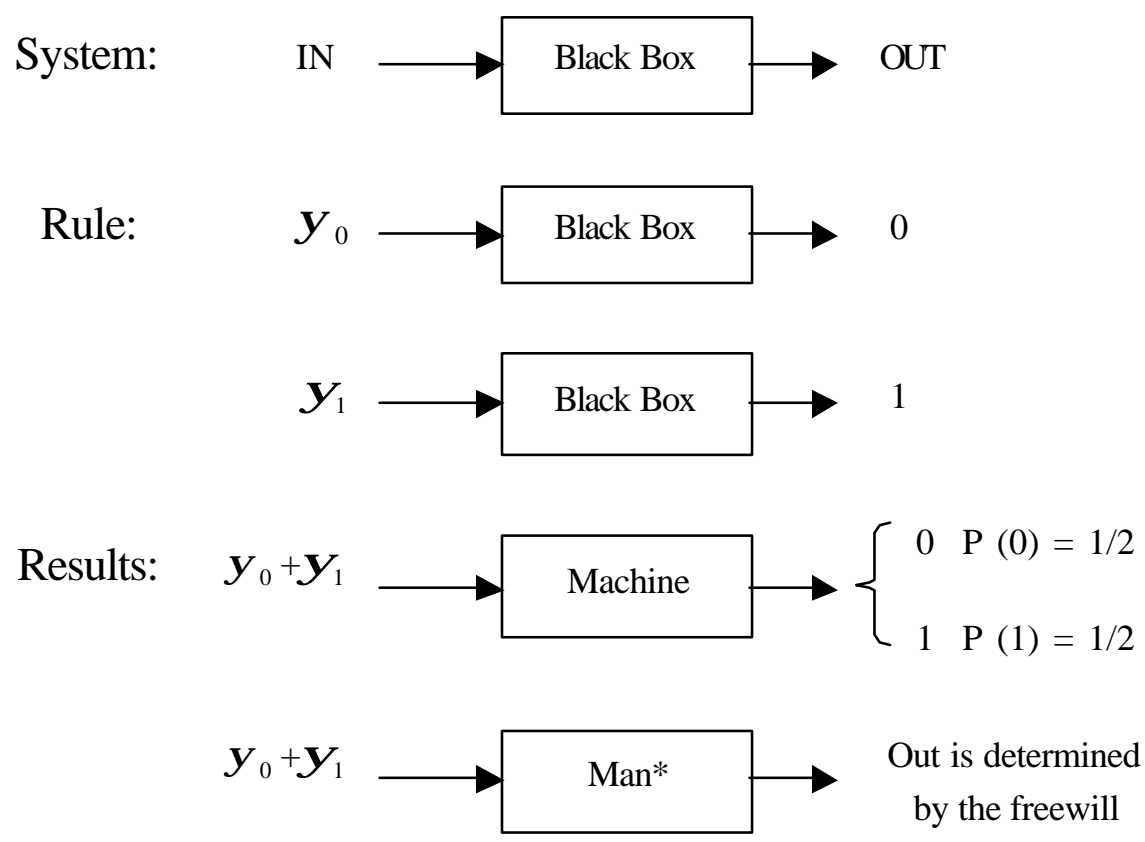

Note: The symbol '*' denotes the conscious being who satisfies the above unusual condition.

\section{Consciousness is a fundamental property of matter}

In this section, we will further argue that consciousness is a fundamental property of matter, and is not reducible or emergent. This may provide a possible quantum basis for panpsychism.

As we have demonstrated, the conscious being, or the matter with consciousness can distinguish the non-orthogonal single states, while the usual physical measuring device, or the matter without consciousness can't. This seems to be also possible if consciousness is reducible or emergent, but there exists an essential difference here. If consciousness is reducible or emergent, then the matter with consciousness must also follow the basic physical principles such as the principle of energy conservation etc. As we know, revised quantum dynamics, which is the promising complete quantum theory, is the most basic physical principle, according to which the non-orthogonal single states can't be distinguished using the usual physical measuring device. But when considering the matter with consciousness, the non-orthogonal single states can be distinguished in principle, and then consciousness evidently violates the basic physical principle. Thus consciousness should be not reducible or emergent, but a new fundamental property of matter.

On the other hand, if consciousness is a new fundamental property of matter, then it is very natural that it violates the present basic physical principle, since the principle doesn't include it as one 
fundamental property of matter. It is expected that a complete theory of matter must describe all properties of matter, thus consciousness, the new fundamental property of matter, must enter the theory from the start. This is undoubted a big challenge for present science.

\section{Some further discussions}

As we know, the most severe problem of panpsychism is the apparent lack of evidence that the fundamental entities of the physical world such as electrons and protons etc possess any consciousness features. Certainly, such "no evidence " argument can be reasonably disputed by noting that there may not exist any signs of complex consciousness at the simplest level, and it may be very difficult to see them even when they do exist there. The existence of gravitation is a good example. Its extreme weakness between the fundamental entities doesn't disconfirm that gravitation is not a fundamental feature of the physical world ( Seager et al, 2001 ).

On the other hand, the above argument, especially the distinguishability of non-orthogonal single states may be used to define the fundamental consciousness property of matter, and further provide some feasible empirical tests to determine whether the fundamental entities of the physical world such as electrons and protons etc possess the consciousness features. Concretely speaking, since the distinguishability of non-orthogonal single states evidently violates the basic linear superposition principle of wave function, the consciousness property of matter will introduce a new kind of non-linearity to the complete evolution equation of wave function, and this new kind of non-linearity is definite, not stochastic. Thus we may use the definite non-linearity element in the complete evolution equation of matter to define the consciousness property of matter. Then just like the other properties of matter such as mass and charge etc, consciousness is also a fundamental property which can be described in mathematics to some extent. Since the existence of such definite non-linearity element can be experimentally tested even for the evolution of the fundamental entities such as electrons and protons etc, the above argument may also provide a well-grounded and promising way to confirm the panpsychism doctrine.

\section{Conclusions}

We show that consciousness may violate the basic quantum principle, according to which the nonorthogonal single states can't be distinguished. The possible implications of the unusual conclusion are deeply analyzed. We demonstrate that it strongly indicates that the physical world is not causally closed without consciousness, and consciousness is a fundamental property of matter. This provides a possible quantum basis and feasible test for panpsychism.

\section{References}

Chalmers, D. (1996), The Conscious Mind (Oxford, University of Oxford Press)

Seager, W. and Allen-Hermanson, S. (2001), Panpsychism, Stanford Encyclopedia of Philosophy

Ghiradi,G.C, Rimini A. and Weber, T. (1986), 'Unified dynamics for microscopic and macroscopic systems', Phys. Rev. D, 34, pp.470-491.

Pearle, P. (1989), 'Combining stochastic dynamical state-vector reduction with spontaneous localization’, Phys. Rev. A 39, pp.2277- 2289.

Diosi, L. (1989), 'Models for universal reduction of macroscopic quantum fluctuations', Phys. Rev. A, 40, pp. 1165-1174. 
Ghiradi,G.C, Rimini A. and Weber, T. (1990), 'A Continuous-spontaneous-reduction model involving gravity', Phys. Rev. D, 42, pp.1057-1064.

Penrose, R. (1996), 'On gravity's role in quantum state reduction', Gen. Rel. and Grav, 28, pp.581-600.

Gao Shan (1999a), 'The collapse problem can be tackled in terms of new motion of particle', LANL e-print physics/9907002.

Gao Shan (1999b), 'From quantum motion to classical motion-seeking the lost reality', Quantum-Mind Digest, \#1999-121

Gao Shan (2000), Quantum Motion and Superluminal Communication (Beijing, Chinese BT Publishing House) http://www.100megspopup.com/ecuq/index.htm

Penrose, R. (1989), The Emperor's New Mind (Oxford, Oxford University Press)

Penrose, R. (1994), Shadows of the Mind (Oxford, Oxford University Press)

Hameroff, S and Penrose, R. (1996a), 'Orchestrated reduction of quantum coherence in brain microtubules: A model for consciousness', In (S. Hameroff, A. Kaszniak, and A. Scott, eds) Toward a Science of Consciousness. MIT Press.

Hameroff, S. and Penrose, R. (1996b). 'Conscious Events as Orchestrated Spacetime Selections', The Journal of Consciousness Studies, 3 (1), pp. 36-53

Gao Shan (1999c), 'How to realize quantum superluminal communication?', LANL e-print quant-ph/9906116.

Bell, J.S. (1987), Speakable and Unspeakable In Quantum Mechanics, (Cambridge, Cambridge University Press)

Duane, D. and Behrendt, T. (1965), 'Extrasensory electroencephalographic induction between identical twins', Science, 150 , pp.367

Grinberg-Zylberbaum, J, Dalaflor, D., Attie, L. and Goswami A. (1994), 'The Einstein-Podolsky-Rosen Paradox in the Brain: The transferred Potential', Physics Essays, 7, pp.422

McGinn, C. (1999), The Mysterious Flame: Conscious Minds in a Material World (New York, Basic Books)

Ghirardi, G. (1999), 'Quantum superpositions and definite perceptions: envisaging new feasible experimental tests', Phys. Lett. A 262, pp.1-14

Thaheld, F.H. (2000), 'Comment on "Quantum superpositions and definite perceptions: Envisaging new experimental tests"', Phys. Lett. A273, pp.232-234 